\title{
Standard Linear Combination of Incline Vectors
}

\author{
AR. Meenakshi and P. Shakila Banu \\ Department of Mathematics, Karpagam University, Coimbatore - 641021 India
}

\begin{abstract}
We prove that every finite subspace generated by the linearly ordered idempotent elements in an incline has a unique standard basis. This leads to every finite subspace of a regular incline whose elements are all linearly ordered has a unique standard basis and thereby we disprove the result of Cao that is "Every subspace of a finite incline whose idempotent elements are linearly ordered has a unique standard basis". As an application we exhibit that under certain conditions each vector in a finitely generated subspace of a vector space has a unique decomposition as a linear combination of the standard basis vectors.
\end{abstract}

Keywords:incline, regular incline, distributive lattice, basis, standard basis, standard linear combination. MS Classification:16R40,16Y60

\section{Introduction}

Inclines are additively idempotent semirings in which products are less than (or) equal to either factor. The notion of inclines and their applications are described by Cao, Kim and Roush[1]. Kim and Roush have surveyed and outlined algebraic properties of inclines and incline matrices [2]. Inclines are generalization of Boolean and Fuzzy algebra. Recently in [5], it is proved that an element in an incline is regular if and only if it is idempotent, further some characterization of regular elements in an incline are discussed and established that every commutative regular incline is a distributive lattice. In our earlier work [6], we have discussed the consistency of the equation $\mathrm{xA}=\mathrm{b}$, where $\mathrm{A}$ is a matrix and $\mathrm{b}$ is a vector over an incline $£$. We have determined the condition for the existence of the maximum solution of the incline relational equation $\mathrm{x} A=\mathrm{b}$.

In general, a vector in an incline need not be expressible uniquely as a linear combination of its standard basis vectors (refer Example 4.1). In this paper, by using the maximum solution of the incline relational equation, we have discussed when a vector can be expressed as a linear combination of the standard basis vectors. As a special case, we have exhibited that each vector in a regular incline whose elements are all linearly ordered has a unique decomposition as a linear combination of its standard basis vectors, which we call as standard incline linear combination. This includes the result found in [3] as a special case for standard linear combination of a vector over the max-min fuzzy algebra. In section 2, we present some basic definitions, notations and required results on inclines. In section 3, we prove that every finite subspace generated by the linearly ordered idempotent elements in an incline has a unique standard basis. This leads to every finite subspace of a regular incline whose elements are all linearly ordered has a unique standard basis and thereby we disprove the result of Cao, that is, "Every subspace of a finite incline whose idempotent elements are linearly ordered has a unique standard basis" (p.38, [1]). In section 4 , we exhibit that each vector $x \varepsilon \AA^{\mathrm{n}}$ can be expressed uniquely as a linear combination of its standard basis vectors $B=\left(c_{1}, c_{2}, \ldots . . c_{n}\right)$, where $c_{j}=\left(c_{j l}\right.$, $\left.c_{j 2}, . ., c_{j n}\right)$ of a finitely generated subspace of a vector space over an incline under the condition that $c_{j k}$ for $j=1$ to $n$ is comparable with the $\mathrm{k}$ th component of $\mathrm{x}$ and for each $\mathrm{k}$. This leads to the structure of the solution set $\Omega(\mathrm{C}, \mathrm{x})$, where $\mathrm{C}$ is the matrix whose rows are the vectors in the standard basis. The main results in the present paper are the generalization of the results available in the literature $[2,3]$.

\section{Preliminaries}

In this section, we present some basic definitions, notations and required results on inclines.

\section{Definition 2.1}

An incline is a nonempty set $£$ with binary operations addition and multiplication denoted as $(+, \cdot)$ (we usually suppress the 'dot' in $\mathrm{a} \cdot \mathrm{b}$ and write as ab) satisfying the following axioms; for a,b,c $\varepsilon £$.

i) $a+b=b+a$

ii) $\mathrm{a}+(\mathrm{b}+\mathrm{c})=(\mathrm{a}+\mathrm{b})+\mathrm{c} \quad$ : $\quad \mathrm{a}(\mathrm{bc})=(\mathrm{ab}) \mathrm{c}$

iii) $\mathrm{a}(\mathrm{b}+\mathrm{c})=\mathrm{ab}+\mathrm{ac} \quad$ : $\quad(\mathrm{b}+\mathrm{c}) \mathrm{a}=\mathrm{ba}+\mathrm{ca}$

iv) $\mathrm{a}+\mathrm{a}=\mathrm{a}$

v) $\mathrm{a}+\mathrm{ac}=\mathrm{a} \quad \mathrm{c}+\mathrm{ac}=\mathrm{c}$

Let us consider an incline $(\mathfrak{f},+, \cdot)$ with the order relation " $\leq$ " defined as $\mathrm{x} \leq \mathrm{y}<=>\mathrm{x}+\mathrm{y}=\mathrm{y}$ for $\mathrm{x}, \mathrm{y} \varepsilon \mathfrak{f}$. This incline order relation " $\leq$ " has the following properties: 
$\mathrm{x}+\mathrm{y} \geq \mathrm{x}$ and $\mathrm{x}+\mathrm{y} \geq \mathrm{y}$ for any $\mathrm{x}, \mathrm{y} \varepsilon £$

$\mathrm{xy} \leq \mathrm{x}$ and $\mathrm{xy} \leq \mathrm{y}$ for any $\mathrm{x}, \mathrm{y} \varepsilon £$

Definition 2.2

A sub incline of an incline $£$ is a subset closed under the incline operations addition and multiplication.

Definition 2.3

A set $S$ of vectors over an incline $£$ is independent if and only if each element of $S$ is not a linear combination of other elements of $S$, that is, no element $v \varepsilon S$ is a linear combination of elements in $S \backslash v$.

\section{Definition 2.4}

A basis for a subspace $\mathrm{W}$ of $£^{\mathrm{n}}$ is a smallest linearly independent set $\mathrm{S}$ of vectors such that $\langle\mathrm{S}\rangle=\mathrm{W}$, where $\langle\mathrm{S}\rangle$ is the space spanned by the set $\mathrm{S}$.

\section{Definition 2.5}

A basis $\mathrm{C}$ over the incline $£$ is a standard basis if and only if whenever and $\mathrm{a}_{\mathrm{ij}} \varepsilon £$ then $\mathrm{a}_{\mathrm{ii}} \mathrm{c}_{\mathrm{i}}=\mathrm{c}_{\mathrm{i}}$.

$$
\mathrm{c}_{\mathrm{i}}=\sum \mathrm{a}_{\mathrm{ij}} \mathrm{c}_{\mathrm{j}} \text { for } \mathrm{c}_{\mathrm{i}}, \mathrm{c}_{\mathrm{j}} \varepsilon \mathrm{C}
$$

\section{Definition 2.6}

a $\varepsilon £$ is said to be regular if there exists an element $\mathrm{x} \varepsilon £$ such that axa $=\mathrm{a}$. Then $\mathrm{x}$ is called a $\mathrm{g}$ - inverse of $\mathrm{a}$ and $\mathrm{a}\{1\}$ denotes the set of all $\mathrm{g}$-inverses of $\mathrm{a}$.

An incline $£$ is regular if and only if each element of $£$ is regular.

\section{Proposition 2.1 [5]}

An element a $\varepsilon £$ is regular if and only if a is idempotent.

\section{Proposition $2.2[5]$}

A commutative incline $£$ is regular if and only if $£$ is a distributive lattice.

Let $£_{\mathrm{mn}}$ and $\mathfrak{£}^{\mathrm{n}}$ denotes the set of all mxn matrices and the set of all $\mathrm{n}$ vectors over $£$ respectively. Let DL be the set of all idempotent elements in $£$. $\mathrm{DL}_{\mathrm{mn}}$ and $\mathrm{DL}^{\mathrm{n}}$ be the set of all mxn matrices and the set of all $\mathrm{n}$ vectors in DL respectively. Let $A_{* j}$ denotes the jth column of $A, N_{r}$ denotes the set of all positive integers 1 to $r$ and $\Omega(\mathrm{A}, \mathrm{b})$ denotes the solution set of the incline relational equation $\mathrm{xA}=\mathrm{b}$. Matrix operations in $£_{\mathrm{nn}}$ are induced by the incline operations of $£$. In general, $\mathfrak{n}_{\mathrm{nn}}$ is not an incline since for any pair of matrices $A, B \varepsilon £_{n n}$, $\mathrm{AB} \leq \mathrm{A}$ (or) $\mathrm{AB} \leq \mathrm{B}$ need not hold.

\section{Standard Bases}

In this section, we prove that every finite subspace of $\mathrm{DL}^{\mathrm{n}}$ over a finite incline $£$ whose idempotent elements are linearly ordered has a unique standard basis. As a special case we deduce that every finite subspace of a regular incline whose elements are all linearly ordered has a unique standard basis and we disprove the result of Cao, that is "Every subspace of a finite incline whose idempotent elements are linearly ordered has a unique standard basis " $\quad$ (p. $38,[1])$

\section{Lemma 3.1}

In a vector space over a finite incline, let $\mathrm{x}=\sum \mathrm{c}_{\mathrm{i}} \mathrm{x}$. Then for some $\mathrm{n}, \mathrm{x}=\sum \mathrm{c}_{\mathrm{i}}{ }^{\mathrm{n}} \mathrm{x}$ where $\mathrm{c}_{\mathrm{i}}^{\mathrm{n}}$ are idempotents.

Proof

Since $\mathrm{x}=\sum \mathrm{c}_{\mathrm{i}} \mathrm{x}=\mathrm{cx}$, where $\mathrm{c}=\sum_{i=1}^{k} c_{i}$ then $\mathrm{c} \geq \mathrm{cx} \geq \mathrm{c}(\mathrm{cx}) \geq \mathrm{c}^{2}(\mathrm{cx}) \geq \ldots . \mathrm{c}^{\mathrm{k}} \mathrm{x} \geq=\ldots$ By using incline property (2.2), we get $c \geq c^{2} \geq c^{3} \geq \ldots \geq c^{n} \geq \ldots$ For a finite incline, $c^{n}$ is idempotent for some $n$.

$$
\text { ...(3.1) }
$$

Again by using incline property $(2.1), \mathrm{c} \geq \mathrm{c}_{\mathrm{i}}$ for each $\mathrm{i}=1$ to $\mathrm{k}$. So we have $\mathrm{c} \geq \mathrm{c}_{\mathrm{i}} \geq \mathrm{c}_{\mathrm{i}}^{2} \geq \ldots \geq \mathrm{c}_{\mathrm{i}}^{\mathrm{m}} \ldots$

For a finite incline $\mathrm{c}_{\mathrm{i}}^{\mathrm{mi}}$ is idempotent for some $\mathrm{m}_{\mathrm{i}}$ and for each $\mathrm{i}=1$ to $\mathrm{k}$. ...(3.2) From (3.1) we get $c^{\mathrm{n}}=\mathrm{c}^{2 \mathrm{n}}$ and from (3.2), $\mathrm{c}_{1}{ }^{\mathrm{ml}}=\mathrm{c}_{1}{ }^{2 \mathrm{ml}}, \mathrm{c}_{2}{ }^{\mathrm{m} 2}=\mathrm{c}_{2}{ }^{2 \mathrm{~m} 2}, \ldots \mathrm{c}_{\mathrm{k}}{ }^{\mathrm{mk}}=\mathrm{c}_{\mathrm{k}}{ }^{2 \mathrm{mk}}$ and $\mathrm{c} \geq \mathrm{c}_{\mathrm{i}}^{\mathrm{mi}}$ for each $\mathrm{i}=1$ to $\mathrm{k}$. Choose the power of $\mathrm{c}$ which is the maximum of $\mathrm{m}_{\mathrm{i}}$ for each $\mathrm{i}=1$ to $\mathrm{k}$.

Hence $\mathrm{x}=\sum \mathrm{c}_{\mathrm{i}}^{\mathrm{n}} \mathrm{x}$, where $\mathrm{c}_{\mathrm{i}}^{\mathrm{n}}$ is idempotent for some $\mathrm{n}>1$. 


\section{Lemma 3.2}

Let $\mathrm{x}$ be a member of a standard basis of $\mathrm{DL}^{\mathrm{n}}$ over a finite incline $£$ whose idempotent elements are all linearly ordered. If $\mathrm{x}=\sum \mathrm{y}_{\mathrm{i}}$ for $\mathrm{y}_{\mathrm{i}}$ in the space spanned by the standard basis of $\mathrm{DL}^{\mathrm{n}}$ then $\mathrm{x}=\mathrm{y}_{\mathrm{k}}$ for some $\mathrm{k}$.

\section{Proof}

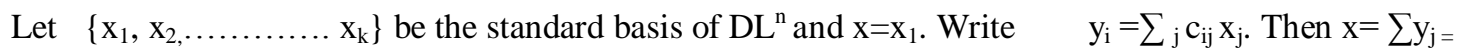
$\sum_{j}\left(\sum_{i} c_{i j}\right) x_{j}$ and by Lemma (3.1), we get $x=\sum c_{i 1}{ }^{n} x$, where $c_{i 1}{ }^{n}$ are idempotents. Since the elements of DL are linearly ordered, let $c_{j 1}{ }^{n}$ be the largest. Then $x=c_{j 1}{ }^{n} x$. By using incline properties (2.1) and (2.2), $=\sum \mathrm{y}_{\mathrm{i}}=>\mathrm{x} \geq \mathrm{y}_{\mathrm{j}}$ and $\mathrm{y}_{\mathrm{i}}=\sum \mathrm{c}_{\mathrm{ij}} \mathrm{x}_{\mathrm{j}}=>\mathrm{x} \geq \mathrm{y}_{\mathrm{j}} \geq \mathrm{c}_{\mathrm{j} 1}{ }^{\mathrm{n}} \mathrm{x}=\mathrm{x}$. Hence, $\mathrm{x}=\mathrm{y}_{\mathrm{j}}$

\section{Theorem 3.1}

Let $£$ be an incline whose idempotent elements are all linearly ordered then any finite subspace of $\mathrm{DL}^{\mathrm{n}}$ has a unique standard basis.

Proof

Let $\left\{\mathrm{x}_{1}, \mathrm{x}_{2} \ldots \ldots \ldots \ldots \mathrm{x}_{\mathrm{k}}\right\}$ and $\left\{\mathrm{y}_{1}, \mathrm{y}_{2} \ldots \ldots \ldots \mathrm{y}_{\mathrm{j}}\right\}$ be the distinct standard basis of a finite subspace of $D L^{n}$. For any $x_{i}$, we have $x_{i}=\sum c_{i k} y_{k}$ for some $c_{i j}$. Then by Lemma (3.2), $x_{i}=c_{i k} y_{k}$ for some $k$. In the same manner, $y_{k}=d_{k j} x_{j}$ for some $j$. If $i \neq j$, then $x_{i}$ would be dependent. Hence, $x_{i}=c_{i k} y_{k}=c_{i k} d_{k i} x_{i}$ by Lemma (3.1), $c_{i k}{ }^{n} d_{k j}{ }^{n} X_{i}=x_{i}$. This proves the uniqueness of the standard basis.

Remark 3.1

In the above Theorem (3.1), the condition that any subspace of $\mathrm{DL}^{\mathrm{n}}$ is essential. This is illustrated in the following example.

\section{Example 3.1}

Let us consider the incline $£=\{0, a, b, c, d, 1\}$, lattice ordered by the following Hasse graph.

Define $£ \mathrm{x} £->£$ as follows

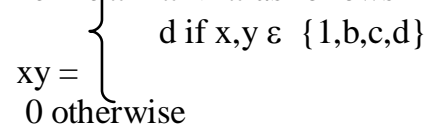

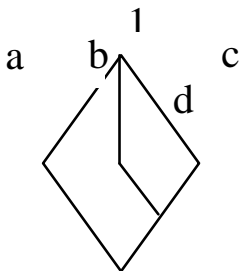

In this finite incline, the only idempotent elements are 0 and $d$ and they are comparable. Since $0=\alpha . d$ and $d=\beta . d$ for $\alpha, \beta \varepsilon £$; (d) is the unique standard basis for $D L=\{0, d\}$. Here, (d) is not a basis for the subincline $\mathrm{I}=\{0, \mathrm{a}, \mathrm{b}, \mathrm{d}, 1\}$.

For instance, $\mathrm{b} \varepsilon \mathrm{I}, \mathrm{b} \neq \alpha \mathrm{d}$ for all $\alpha \varepsilon £$, therefore $\mathrm{b}$ cannot be expressed as a linear combination of the elements of $£$.

This contradicts the Theorem (2.4.4) of [1].

\section{Remark 3.2}

Further, in a regular incline $£$ by proposition (2.1) each element of $£$ is idempotent, hence DL $=£$. And by proposition (2.2), every commutative regular incline is a distributive lattice. Hence we deduce the following as special cases of Theorem (3.1).

\section{Corollary 3.1}

basis.

Every finite subspace of a regular incline whose elements are all linearly ordered has a unique standard

\section{Corollary 3.2} standard basis.

Every finite subspace of a distributive lattice whose elements are all linearly ordered has a unique

\section{Remark 3.3}

For a fuzzy algebra with support $[0,1]$ under the operation max-min

(or min-max) the elements are all idempotents and linearly ordered. Hence Theorem (3.1) reduces to the result of Kim and Roush [3] (P.8, [4])

\section{Corollary 3.3}

Any finitely generated subspace of a fuzzy algebra has a unique standard basis. 


\section{Standard linear combination of incline vectors}

In this section, we exhibit that each incline vector has a unique decomposition as a linear combination of the standard basis of a finitely generated subspace of a vector space over a regular incline whose elements are all linearly ordered.

For $\mathrm{x}, \mathrm{y} \varepsilon £$, if $\mathrm{x} \leq \mathrm{y}$ for all $\mathrm{y} \varepsilon £$ then $\mathrm{x}$ is called the least element and denoted as 0 . If $\mathrm{x} \geq \mathrm{y}$ for all $\mathrm{y} \varepsilon £$ then $\mathrm{x}$ is called the greatest element and denoted as 1 .

\section{Lemma 4.1}

Proof

For a regular incline $£$, the greatest element 1 coincides with the multiplicative identity of $£$.

Let a $\varepsilon £$, then a is idempotent. By incline property (2.1),

$$
\mathrm{a}=\mathrm{a}^{2}=\mathrm{a} . \mathrm{a} \leq \mathrm{a} \cdot 1 \leq
$$
$\mathrm{a}=>\mathrm{a}=\mathrm{a} .1$. Similarly, $\mathrm{a}=\mathrm{a}^{2}=\mathrm{a} \cdot \mathrm{a} \leq 1 . \mathrm{a} \leq \mathrm{a}=\mathrm{a}=1 \mathrm{a}$. Thus, a. $1=1 . \mathrm{a}=\mathrm{a}$ for all a $\varepsilon £$. Hence 1 is the multiplicative identity of $£$.

In this section, to determine the standard linear combination of incline vector we shall make use of the following result found in [6].

\section{Lemma 4.2}

Let $\mathrm{xA}=\mathrm{b}$ be the incline relational equation with $\mathrm{x}=\left[\mathrm{x}_{\mathrm{j}} / \mathrm{j} \varepsilon \mathrm{N}_{\mathrm{m}}\right], \mathrm{b}=\left[\mathrm{b}_{\mathrm{k}} / \mathrm{k} \varepsilon \mathrm{N}_{\mathrm{n}}\right]$ and $\mathrm{A}=\left(\mathrm{a}_{\mathrm{ij}}\right) \varepsilon £_{\mathrm{mn}}$ such that $\mathrm{A}_{*_{\mathrm{k}}}$ and $\mathrm{b}_{\mathrm{k}}$ are comparable for each $\mathrm{k}$. Then $\Omega(\mathrm{A}, \mathrm{b}) \neq \varphi$ if and only if $\hat{\mathrm{x}}=\left[\hat{\mathrm{x}}_{j} / j \varepsilon \mathrm{N}_{\mathrm{m}}\right]$ defined as $\hat{\mathrm{x}}_{\mathrm{j}}=\min \sigma$ $\left(\mathrm{a}_{\mathrm{jk}}, \mathrm{b}_{\mathrm{k}}\right)$

is the maximum solution.

$$
\text { Where } \sigma\left(\mathrm{a}_{\mathrm{jk}}, \mathrm{b}_{\mathrm{k}}\right)=\left\{\begin{array}{cc}
b_{k} & \text { if } \\
1 & \text { otherwise }
\end{array} \mathrm{a}_{j k}>b_{k}\right\}
$$

\section{Theorem 4.1}

Let $£$ be an incline, $\mathrm{S}$ be a finitely generated subspace of $£^{\mathrm{n}}$ and let $\left\{\mathrm{c}_{1}, \mathrm{c}_{2}, \ldots \ldots \ldots \mathrm{c}_{\mathrm{n}}\right\}$ be the standard basis for $S$. For $x \varepsilon S$ and $c_{j}=\left\{c_{j 1}, c_{j 2}, \ldots c_{j k}, \ldots c_{j n}\right\}$, if $c_{j k}$ and $x_{k}$ are comparable for each $j=1$ to $n$ and each $k$. Then $\mathrm{x}$ can be expressed uniquely as a linear combination of the standard basis vectors.

Proof

Since $\left\{\mathrm{c}_{1}, \mathrm{c}_{2}, \ldots \ldots \ldots \mathrm{c}_{\mathrm{n}}\right\}$ is a standard basis for $\mathrm{S}, \mathrm{x}$ is a linear combination of the standard basis vectors. Let $\mathrm{x}=\sum_{j=1}^{n} \beta_{\mathrm{j}} \mathrm{c}_{\mathrm{j}}$ Where $\beta_{\mathrm{j}} \varepsilon £$.

In this expression, the coefficients $\beta_{\mathrm{j}}$ 's are not unique. If we write this in the matrix form as $\mathrm{x}=\left(\beta_{1}\right.$, $\left.\beta_{2}, \ldots \ldots \ldots \beta_{\mathrm{n}}\right) \mathrm{C}$, where $\mathrm{C}$ is the matrix whose rows are the basis vectors, then $\mathrm{x}=\mathrm{P} \mathrm{C}$ has a solution, that is, $\Omega$ $(\mathrm{C}, \mathrm{x}) \neq \Phi$ since the kth column of $\mathrm{C}=\mathrm{C}_{* \mathrm{k}}$, is comparable with the kth component of $\mathrm{x}$ for each $\mathrm{k}$; by using Lemma (4.2), it follows that this equation has a unique maximum solution $\quad \hat{\mathrm{x}}=\left(p_{1}, p_{2}, \ldots \ldots \ldots p_{\mathrm{n}}\right)$ (say). Then $\mathrm{x}=\sum_{j=1}^{n} p_{\mathrm{j}} \mathrm{c}_{\mathrm{j}}$ where $p_{\mathrm{j}} \varepsilon £$ is the unique representation of the vector $\mathrm{x}$, we call this representation as the standard linear combination of the vector $\mathrm{x} \varepsilon £^{\mathrm{n}}$

\section{Remark 4.1}

In the above Theorem (4.1), the condition that the kth column of $\mathrm{C}$ whose rows are the basis vectors and the kth component of $\mathrm{x}$ to be comparable is essential in determining the standard linear combination of incline vector is illustrated in the following.

\section{Example 4.1}

Let us consider the set $D=\{a, b, c\}$ and the incline $£=(P(D), \cup, \cap)$, where $P(D)$ is the power set of $D$ with set inclusion " $\subseteq$ " as the order relation " $\leq$ ".

Here, $£$ is a commutative regular incline hence by proposition (2.2), $£$ is a distributive lattice whose elements are all idempotents; but elements are not comparable. For instance, $\{a\},\{b\}$ and $\{c\}$ are not comparable.

Consider the set $S=(\{a\},\{b\},\{c\})$. In $S$ each element cannot be expressed as a linear combination of the remaining elements. Hence $S$ is the smallest linearly independent set and $\langle S\rangle=£$. Every element of $£$ can be expressed as a linear combination of the basis vectors. For $\{a\} \varepsilon £,\{a\}=\alpha\{a\}+\beta\{b\}+\gamma\{c\}, \alpha$ is any subset of $D$ containing $\{a\}, \beta$ is any subset of $D$ not containing $\{b\}$ and $\gamma$ is any subset of $D$ not containing $\{c\}$. Thus $\{\mathrm{a}\}=\alpha\{\mathrm{a}\}$. Hence $\mathrm{S}$ is the unique standard basis for $£$.

Any element $\mathrm{x} \neq \mathrm{D} \varepsilon £$ can be expressed as $\mathrm{x}=\alpha\{\mathrm{a}\}+\beta\{\mathrm{b}\}+\gamma\{\mathrm{c}\}$ for suitable choice of $\alpha, \beta, \gamma \varepsilon £$. However, this expression is not unique in the sense that the coefficients of $\alpha, \beta$ and $\gamma$ are not uniquely determined by $\mathrm{x}$, since the kth component of $\mathrm{x}$ is not comparable with the kth columnof $\mathrm{C}=$

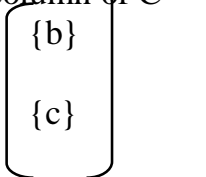


In this incline, $\mathrm{x}=\mathrm{D}=\{\mathrm{a}, \mathrm{b}, \mathrm{c}\}$ is the only element of $£$ which is comparable with the column matrix $\mathrm{C}$. Hence, by Theorem (4.1), D is the only element in this incline which has the standard linear combination. We can find the standard linear combination of the element $\mathrm{D} \varepsilon £$, by determining the maximum solution of the incline relational equation $\mathrm{yC}=\mathrm{x}$. From Lemma (4.2), the maximum solution $\hat{\mathrm{y}}=\left(\hat{\mathrm{y}}_{1}, \hat{\mathrm{y}}_{2}, \hat{\mathrm{y}}_{3}\right)$ is determined by $\hat{\mathrm{y}}_{\mathrm{j}}=$ $\min _{\varepsilon} \sigma\left(\mathrm{c}_{\mathrm{j} k}, \mathrm{x}_{\mathrm{k}}\right)$. Here, $\mathrm{x}=\mathrm{D}=\{\mathrm{a}, \mathrm{b}, \mathrm{c}\}$ is the greatest element of $£$ under the set inclusion as order relation; $\mathrm{c}_{11}=$ $\{a\}, c_{21}=\{b\}$ and $c_{31}=\{c\}$, therefore, $\hat{y}_{1}=\sigma\left(c_{11}, x\right)=1,, \hat{y}_{2}=\sigma\left(c_{21}, x\right)=1$, and $\hat{y}_{3}=\sigma\left(c_{31}, x\right)=1$. Hence, $\hat{y}=$ $(1,1,1)=(\mathrm{D}, \mathrm{D}, \mathrm{D})$. Thus $\mathrm{D}=\mathrm{D}\{\mathrm{a}\}+\mathrm{D}\{\mathrm{b}\}+\mathrm{D}\{\mathrm{c}\}$ is the standard linear combination of the element $\mathrm{D} \varepsilon \mathrm{f}$.

\section{Conclusion}

We have discussed the uniqueness of the standard basis and standard linear combination of incline vectors. In a finitely generated subspace $£^{\mathrm{n}}$ of a regular incline whose elements are all linearly ordered, the comparability condition automatically holds, hence we conclude that each vector in a regular incline whose elements are all linearly ordered has a standard linear combination. The main results in the present paper are the generalization of the results found in [2] and [3].

\section{Acknowledgement}

I thank to my Guide Dr. AR.Meenakshi, Former Emeritus Professor of Mathematics, Annamalai University, Chidambaram for her guidance.

\section{References}

[1] Z.Q. Cao , K.H, Kim, F.W. Roush, Incline algebra and applications, John Wiley and Sons, New York, 1984.

[2] K.H. Kim, F.W. Roush, Inclines and incline matrices: a survey, Linear algebra appl., 379,457-473(2004).

[3] K.H.Kim, F.W.Roush, Generalized fuzzy matrices, Fuzzy sets and systems, 4, 293-315 (1980).

[4] AR.Meenakshi, Fuzzy matrix Theory and its applications, MJP Publishers, Chennai, 2008.

[5] AR.Meenakshi, S. Anbalagan, On regular elements in an incline, Int J. Math. and Math. Sci. (2010) article ID 903063, 12 Pages.

[6] AR.Meenakshi, P.Shakila Banu, Incline relational equations (communicated). 